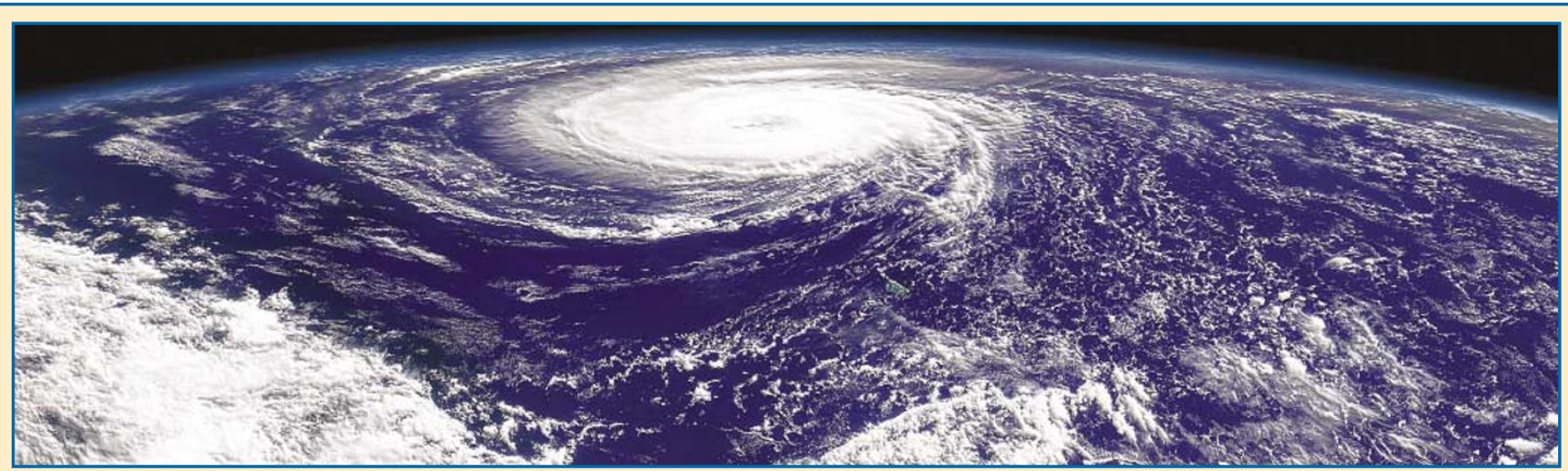

Meteorology

\title{
Getting the wind up
}

Once research into global change

took off on a large scale, few

phenomena related to climate escaped measurement. Researchers have dragged unwieldy equipment up mountains in the tropics to drill into the glaciers found there. And the deep ocean has been probed by fleets of sophisticated floats. But wind speeds in the eyewall of hurricanes, where the storm is at its most intense, have long defied observation. On page 279 of this issue, however, Mark Powell and his colleagues present just such measurements (Nature 422, 279-283; 2003).

The data -331 vertical wind profiles from 15 tropical cyclones were obtained by dropwind sondes from the Global Positioning System. The sondes are launched from aircraft at altitudes of 1.5-3 km; they relay their position and measure pressure, temperature and humidity as they fall through the atmosphere and drift with the wind. Tropical cyclones - such as Hurricane Alberto, which occurred in August 2000 and is shown in the satellite image here - derive much of their energy from sea-air exchange. For forecasting purposes it is essential to have accurate information about relevant parameters such as the exchange of momentum, which partly depends on the drag factor exerted by the state of the sea surface.

One of Powell and colleagues' findings is that maximum wind speeds occur at an altitude of about $500 \mathrm{~m}$. More notably, however, they find that one assumption used in predicting the intensity and consequences of hurricanes is incorrect. Previously, in the absence of observations for wind speeds above $25 \mathrm{~m} \mathrm{~s}^{-1}$, levels of increasing drag with increasing wind speed were extrapolated to high wind speeds. But now it seems that above hurricane force - about $33 \mathrm{~m} \mathrm{~s}^{-1}$ - a layer of foam and bubbles from breaking waves develops that reduces drag and effectively lets the hurricane glide over the sea. In consequence, air-sea exchange in hurricanes will need to be reassessed.

Heike Langenberg as several transcription factors ${ }^{2}$. Moreover, hair-follicle development is severely impaired when the noggin gene, which encodes a BMP inhibitor, is deleted ${ }^{5}$. Jamora and colleagues now link BMP and Wnt signalling by showing that activation of the Wnt pathway results in the formation of $\beta$-catenin-activated Lef1 transcription complexes - and consequently in decreased E-cadherin expression - only when combined with the inhibition of BMP signalling by noggin ${ }^{1}$.

So is the downregulation of E-cadherin indeed necessary for hair-follicle formation? Cadherins are calcium-dependent proteins that mediate cell-cell adhesion, often as part of complexes called adherens junctions, and they are known to be involved in development and tissue maintenance ${ }^{6}$. In the skin, E-cadherin is expressed in the developing epidermis (whereas P-cadherin becomes predominant later in the growing hair follicle $)^{7}$. Previous work has shown that the loss of some intracellular cadherin-binding proteins severely impairs hair-follicle formation ${ }^{4,8}$, but no one had looked at whether E-cadherin itself is required in this process. Jamora et al. now test this and show that the forced expression of E-cadherin in the developing skin results in increased adhesiveness and abnormal epithelial buds. So, the loss of E-cadherin (and, by implication, changes in adherens junctions) appears to be crucial for epithelial buds to begin forming. This supports a model in which downregulation of the E-cadherin gene in stem cells that receive noggin and Wnt signals facilitates cell movement, rearrangement and bud formation. It is also consistent with previous observations that the loss of Ecadherin expression or function can lead to enhanced invasive behaviour of other cells ${ }^{6,9}$.

Together with previous work ${ }^{10}$, the results of Jamora and colleagues provide strong evidence that dynamic changes in the composition of adherens junctions are important for the development of skin appendages such as hair follicles. The results may also be relevant to the development of other tissues and organs from epithelial buds (for example, teeth, lungs and limbs). That said, many questions remain unanswered. For instance, the pelage follicles (guard hairs) that occur in mice develop in a Lef1-independent manner. Yet the new results imply that downregulation of E-cadherin expression is common to all developing hair follicles. This hints that other transcription regulators can also repress E-cadherin expression in epithelial cells during follicle formation (and we know of good candidates, such as members of the Snail family $\left.{ }^{11,12}\right)$. In addition, epithelial buds do not develop randomly, but follow a precise geometrical pattern. What governs this?
Another important question is what determines whether an epithelial bud contributes to a hair follicle, sweat gland or mammary gland. Here there are likely to be species-specific mechanisms, because we know, for example, that hair follicles and sweat glands form in mutually exclusive regions in mice, whereas in humans both cell types can develop from neighbouring stem cells. But are these fates specified early in bud formation or later during bud expansion? The choices an epithelial bud makes are clearly intricate and await investigation.

Yann Barrandon is at the School of Life Sciences, Swiss Federal Institute of Technology (EPFL), and the Department of Experimental Surgery, Vaud University Hospital (CHUV),

1011 Lausanne, Switzerland.

e-mail: yann.barrandon@epfl.ch

1. Jamora, C., DasGupta, R., Kocieniewski, P. \& Fuchs, E. Nature 422, 317-322 (2003).

2. Fuchs, E. \& Raghavan, S. Nature Rev. Genet. 3, 199-209 (2002). 3. Eastman, Q. \& Grosschedl, R. Curr. Opin. Cell Biol. 11, 233-240 (1999).

4. Huelsken, J. et al. Cell 105, 533-545 (2001).

5. Botchkarev, V. A. et al. Nature Cell Biol. 1, 158-164 (1999).

6. Takeichi, M. Science 251, 1451-1455 (1991).

7. Hirai, Y. et al. Development 105, 271-277 (1989).

8. Vasioukhin, V. et al. Cell 104, 605-617 (2001)

9. Gumbiner, B. M. J. Cell Biol. 148, 399-403 (2000).

10. Perez-Moreno, M., Jamora, C. \& Fuchs, E. Cell 112, 535-548 (2003)

11. Cano, A. et al. Nature Cell Biol. 2, 76-83 (2000). 12. Batle, E. et al. Nature Cell Biol. 2, 84-89 (2000). 